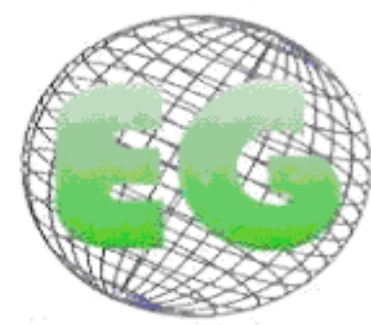

ISSN 1695-6141

$\mathrm{N}^{\circ} 24$
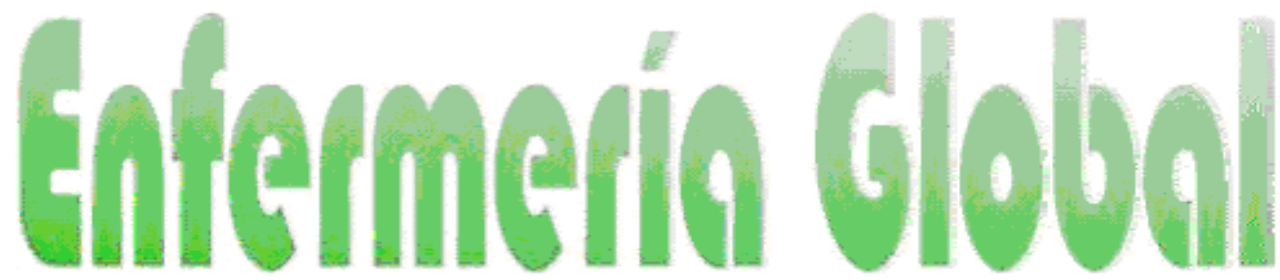

Revista electrónica trimestral de Enfermería

Octubre 2011

www.um.es/egloball

\title{
Aparición de complicaciones relacionadas con el uso del catéter venoso central de inserción periférica (PICC) en los recién nacidos
}

Ocorrência de complicações relacionadas ao uso de Cateter Venoso Central de Inserção Periférica (PICC) em recém-nascidos

\begin{abstract}
${ }^{*}$ Montes, SF., ${ }^{* *}$ Teixeira, JBA., ${ }^{* * *}$ Barbosa, MH., ${ }^{* * *}$ Barichello, E.
*Enfermeira Graduada. **Mestre em Enfermagem. Professora Assistente do CGE da UFTM. Universidade Federal do Triângulo Mineiro (UFTM). ${ }^{* * *}$ Doutora em Enfermagem na Saúde do Adulto. Professora Adjunta do CGE da UFTM. Uberaba-MG, Brasil.
\end{abstract}

Palabras clave: Recién nacidos; Catéter de demora; Enfermería.

Palavras-chave: Recém nascidos; Cateter de demora; Enfermagem.

Keywords: Newborn; Permanent catheters; Nursing

\section{RESUMEN}

Este estudio retrospectivo tuvo como objetivo identificar la aparición de complicaciones asociadas al catéter central de inserción periférica (PICC) en recién nacidos (RN) en la Unidad de Cuidados Intensivos Neonatales de octubre de 2008 a julio de 2009, en un hospital público de enseñanza de Uberaba, Minas Gerais. Se realizaron 55 procedimientos para la inserción del PICC en $41 \mathrm{RN}$ que fueron hospitalizados en este período, con un promedio de 1,3 procedimientos de inserción por RN. Se evidenció que la mayoría de los RN tenían un peso bajo al nacer $(1.639,5 \pm 632,7 \mathrm{~g})$ con una edad media gestacional de $31,9 \pm 4,1$ semanas. Se verificó la inserción del PICC en $15(27,3 \%)$ en la vena safena, $13(23,6 \%)$ en la cabeza y $10(18,2 \%)$ en la basílica. El promedio de intentos de punción fue de 3,1 veces por procedimiento. A los Rayos X, 21 (38,2\%) se encontraban en una ubicación céntrica. Se observó 17 (30,9\%) obstrucción de la luz, 9 (16,4\%) de ruptura del catéter y $2(4,9 \%)$, flebitis. La duración media del PICC fue de 8,8 días. Estos hallazgos señalan la necesidad de mayores inversiones en programas de formación profesional para garantizar el manejo seguro de dispositivos intravenosos y asegurar la calidad de la asistencia de enfermería a esta población.

\section{RESUMO}

Estudo retrospectivo que teve como o objetivo identificar a ocorrência de complicações associadas ao cateter central de inserção periférica (PICC) em recém-nascidos (RN) internados na Unidade de terapia Intensiva Neonatal de outubro de 2008 a julho de 2009, em um hospital público de ensino de Uberaba, Minas Gerais. Foram realizados 55 procedimentos de inserção do PICC em $41 \mathrm{RN}$ que estiveram internados neste período, com média de 1,3 procedimentos de inserção por RN. Evidenciou-se que a maioria dos RN apresentava baixo peso $(1639,5 \pm 632,7 \mathrm{~g}) \mathrm{com}$ a média de idade gestacional de $31,9 \pm 4,1$ semanas. Verificou-se a inserção do PICC em $15(27,3 \%)$ na veia safena, $13(23,6 \%)$ na cefálica e $10(18,2 \%)$ na basílica. A média de tentativas de punções foi de 3,1 vezes por procedimento. Aos raios-X, 21 (38,2\%) encontravam-se centralmente localizados. Observou-se $17(30,9 \%)$ obstrução do lúmen, $9(16,4 \%)$ ruptura do cateter e $2(4,9 \%)$ flebite. A média de permanência do PICC foi de 8,8 dias. Estes achados apontam para a necessidade de ampliação de 
investimentos em programas de capacitação profissional para garantir o manejo seguro destes dispositivos intravenosos e assegurar a qualidade da assistência de enfermagem a esta população.

\section{ABSTRACT}

This retrospective study aimed to identify the occurrence of complications associated with peripherally inserted central catheter (PICC) in newborns (NB) in the Unit of Intensive therapy Neonatal from October 2008 to July 2009, at a public teaching hospital Uberaba, Minas Gerais. 55 procedures were performed for insertion of the PICC in 41 infants who were hospitalized during the period, averaging 1.3 procedures per incorporation by RN. The results show that the majority of infants had low birth weight $(1639.5 \pm 632.7 \mathrm{~g})$ and with a mean gestational age of $31.9 \pm 4.1$ weeks. The preferred veins for insertion of the PICC were: saphenous with $15(27.3 \%) ; 13$ $(23.6 \%)$ in the cephalic and $10(18.2 \%)$ in the basilica. The average puncture attempts was 3.1 times per procedure. $21(38.2 \%)$ were centrally located by X-rays. Obstruction of the lumen was observed in $17(30.9 \%)$ cases, occurred rupture catheter in 9 (16.4\%), and phlebitis in $2(4.9 \%)$. The mean length of the PICC was 8.8 days. These findings point to the need for greater investments in professional training programs to ensure the safe management of intravenous devices and ensure the quality of nursing care in this population.

\section{INTRODUCCIÓN}

Los avances tecnológicos y el desarrollo de nuevos recursos terapéuticos en neonatología, han proporcionado un notable aumento en la supervivencia de recién nacidos (RNs) gravemente enfermos, especialmente los prematuros y de bajo peso. La terapéutica intravenosa tiene un papel muy importante en la asistencia de estos neonatos que frecuentemente necesitan de medicamentos de uso prolongado. Así, disponer de un acceso venoso seguro y funcional es fundamental para su supervivencia ${ }^{(1)}$.

Actualmente, están disponibles en el mercado diversos tipos de dispositivos intravenosos que pueden ser utilizados en la asistencia de estos recién nacidos. Entre estos, el catéter central de inserción periférica (PICC) es considerado una vía de acceso venoso segura con ventajas y menores tasas de complicaciones si se compara con los catéteres por procedimiento quirúrgico ${ }^{(2)}$.

Hay también otras ventajas del PICC que son citadas, entre ellas la posibilidad de inserción a la cabecera del enfermo realizada por el enfermero, sin necesidad de un procedimiento quirúrgico; menor incidencia de hemorragia y de neumotórax; optimización de la asistencia intravenosa sin interrupción del tratamiento; menor incomodidad del RN; preservación del sistema venoso periférico; menor costo comparado con los dispositivos insertados quirúrgicamente; posibilidad de infusión de nutrición parenteral y soluciones vesicantes por un tiempo prolongado con bajo riesgo de infiltración y flebitis química; menor riesgo de infección en relación a otros dispositivos vasculares centrales; y disminución del estrés del $\mathrm{RN}$ y del equipo de enfermería por punciones repetitivas ${ }^{(3)}$.

La inserción del PICC está indicada en casos en que haya necesidad de terapias de duración prolongada (más de una semana); administración de nutrición parenteral (NP) con concentración de dextrosa mayor de 10\%; infusión de medicamentos vesicantes o irritantes, de soluciones hiperosmolares o con $\mathrm{pH}$ no fisiológico; y cuando fuere necesario monitorizar la presión venosa central ${ }^{(1,2,3)}$.

EI PICC consiste en un dispositivo vascular inserto a través de una vena superficial periférica que avanza por medio de una aguja introductora y con la ayuda del flujo sanguíneo hasta la vena cava superior, donde toma característica de acceso central. EI PICC utilizado en neonatalogía en general es de único lumen debido al pequeño calibre, siendo el más común de 1,9 french. En cuanto al material puede estar constituido de poliuretano o elastómeros de 
silicona, que son materiales biocompatibles, menos trombogénicos y que dificultan la agregación de microorganismos en su pared. El lugar de inserción es una vena periférica preservada, de calibre adecuado y no tortuosa, las más indicadas son la vena basílica y la cefálica $^{(4,5)}$.

El procedimiento puede ser realizado a la cabecera del enfermo, con anestesia local, sin necesidad de un procedimiento quirúrgico; debiendo ser insertado precozmente cuando la red venosa todavía está preservada, una vez que la presencia de edema, eritema, hematoma, o lesión cutánea, causados por punciones venosas anteriores, dificultan la progresión del catéter. Este procedimiento puede ser realizado por un enfermero debidamente capacitado ${ }^{(2,3,4)}$.

Existen contraindicaciones de realizar el procedimiento: en $\mathrm{RN}$ con lesiones cutáneas en el lugar de inserción; en la administración de grandes volúmenes en bolos o bajo presión; cuando el retorno venoso esté deteriorado; en caso de emergencia; y cuando los familiares se niegan a aceptar el procedimiento ${ }^{(2)}$.

EI PICC, como ya se ha explicado anteriormente, puede permanecer largos períodos. Entre las causas de su retirada se pueden citar: fin de la terapia propuesta, retirada accidental, mala posición de la punta del catéter, presencia de complicaciones como: obstrucción, rotura, infección, fiebre y extravasación o incluso óbito del paciente ${ }^{(5)}$.

Entre las desventajas de la utilización del dispositivo encontramos: la necesidad de entrenamiento especial del equipo para la inserción y sostenimiento; acceso de venas periféricas calibrosas; tiempo de inserción de cerca de 45 minutos; necesidad de utilización de curativos que promuevan barrera máxima; y la contraindicación en casos de emergencia $^{(3)}$.

Las tasas de complicaciones asociadas a PICC son menores comparadas con los catéteres por procedimiento quirúrgico. Con todo pueden ocurrir complicaciones graves relacionadas con la introducción del catéter como: derrames pleurales y pericárdicos, taponamiento cardíaco, perforación del miocardio, arritmias cardíacas, desplazamiento del catéter, trombosis vascular, bacteremia, endocarditis, sepsis, embolización pulmonar tras fractura y desplazamiento del catéter ${ }^{(1,5,6)}$.

El profesional enfermero que actúa en la unidad de terapia intensiva neonatal debe estar capacitado para el paso del PICC, su mantenimiento y prevención de complicaciones. Considerado el PICC como una tecnología reciente utilizada en la asistencia de enfermería al RN, este estudio tuvo como objetivo identificar la ocurrencia de complicaciones relacionadas con el PICC en RN internados en una Unidad de terapia intensiva neonatal de un hospital público de enseñanza de Uberaba-MG.

\section{MÉTODO}

Se realizó un estudio retrospectivo, descriptivo, con abordaje cuantitativo, en un hospital de gran tamaño, público, de enseñanza, que atiende a pacientes de alta complejidad del municipio de Uberaba, Minas Gerais.

La población del estudio estuvo compuesta por RNs internados en la UTI Nenonatal en el periodo de octubre de 2008 a julio de 2009 y que fueron sometidos al procedimiento de inserción del PICC. 
Se identificaron $49 \mathrm{RN}$ sometidos a inserción del PICC en el sector de UTI Neonatal. De estos, ocho prontuarios no fueron localizados junto al Servicio de Archivo Médico y Estadística (SAME), siendo excluidos del estudio.

Se analizaron 55 procedimientos de inserción de PICC realizados en los 41 RN que constituían la muestra de este estudio.

Los datos se obtuvieron de las fichas de acompañamiento de los RNs internados en la UTI y de los prontuarios, junto al Servicio de Archivo Médico y Estadística (SAME), del hospital campo de estudio, por la investigadora.

La obtención de los datos fue dirigida por un instrumento que abarca los aspectos clínicos y epidemiológicos de los RNs (sexo, edad gestacional, peso de nacimiento, diagnóstico médico y evolución de caso); las características en cuanto al procedimiento de implantación del PICC (lugar de inserción, vena, número de tentativas de punción y localización de la punta del catéter); las características del procedimiento de eliminación do PICC (motivo de eliminación, cultivo de la punta del catéter, tiempo de permanencia) y datos sobre las complicaciones relacionadas con el PICC presentadas por los RNs (sepsis, bacteremia, infiltración de los solutos perfundidos, cambio, desplazamiento).

La colecta de datos se inició tras la aprobación del proyecto de investigación por la Comissão de Ética e Pesquisa da Universidade Federal do Triângulo Mineiro (UFTM) Parecer n. 1374/09, respetando la Resolución no 196/96 sobre investigaciones que envuelven seres humanos.

Los datos obtenidos se introdujeron en una Planilla electrónica Exce ${ }^{\circledR}$ para Windows $X P^{\circledR}$ para análisis. Se realizó estadística descriptiva, con frecuencias absoluta y relativa, media y desvío patrón y los resultados se presentan en forma de tablas y figuras.

\section{RESULTADOS Y DISCUSIÓN}

En el periodo de octubre de 2008 a julio de 2009, 49 RNs fueron sometidos a inserción del PICC en la institución campo de estudio. De estos, 41 prontuarios fueron localizados y se realizaron 55 procedimientos de inserción de PICC, analizados en este estudio.

En relación a los RN (41) sometidos a los procedimientos de inserción del PICC se verificó que hubo equilibrio entre los sexos, la edad gestacional varió entre 35 a 40 semanas (media de 31,9 $\pm 4,1$ semanas) y el peso al nacer fue de $715 \mathrm{~g}$ a $2826 \mathrm{~g}$ (media de $1639,5 \pm 632,7 \mathrm{~g}$ ), lo que caracterizó una población de prematuros y de bajo peso, según los parámetros del Centers for Disease Control and Prevention ${ }^{(8)}$. Estos hallazgos corroboran con otros estudios que apuntan las mismas características, no obstante el predominio del sexo masculino ${ }^{(6-7,9)}$.

En cuanto a los motivos de ingreso en la UTI neonatal, $25(61,0 \%)$ fueron por alteraciones respiratorias; $7(17,1 \%)$ alteraciones infecciosas (sífilis congénita, toxoplasmosis congénita, sepsis neonatal y choque séptico); 4 (9,8\%) alteraciones metabólicas; 3 (7,3\%) malformaciones congénitas y $2(4,8 \%)$ cardiopatía congénita, también evidenciados en otros estudios ${ }^{(6,10)}$. La mayoría, 39 (87,8\%) evolucionó con alta de esta unidad (UTI neonatal). 
Se realizaron 55 procedimientos de inserción del PICC en los $41 \mathrm{RNs}$, con una media de 1,3 procedimientos por RN. El principal lugar de inserción fue $15(27,3 \%)$ la vena safena (Tabla 1).

En desacuerdo con los resultados encontrados en este estudio, la vena basílica ha sido la más utilizada, como lugar de inserción. Esta opción se da por las características anatómicas favorables, como mayor calibre, menor número de válvulas, además de la localización que facilita la manipulación para el cambio de los curativos. La vena cefálica ha sido la segunda opción, también identificado en esta investigación ${ }^{(13,14,15)}$.

Otros estudios realizados también utilizaron para la inserción del PICC, las venas basílica y cefálica como segunda opción ${ }^{(16-17)}$.

Hay estudios que apuntan mayor tiempo de permanencia del PICC cuando su inserción se dio en las venas de la fosa antecubital, con duración media de la cateterización de $16,4^{(17)}$. En este estudio, la media de duración del PICC fue de 8,8 días, siendo las venas safena y cefálica las más utilizadas para la inserción del catéter.

Tabela 1 - Distribuição relacionada ao local de inserção do cateter PICC. Uberaba (MG) -2009 .

\begin{tabular}{lcc}
\hline Veias & $\mathbf{N}=\mathbf{5 5}$ & $\mathbf{\%}$ \\
\hline Safena & 15 & $\mathbf{2 7 , 3}$ \\
Cefálica & 13 & 23,6 \\
Basílica & 10 & 18,2 \\
Axilar & 7 & 12,7 \\
Jugular & 6 & 10,9 \\
Femoral & 2 & 3,7 \\
Retroauricular & 1 & 1,8 \\
Não informado & 1 & 1,8 \\
\hline TOTAL & $\mathbf{5 5}$ & $\mathbf{1 0 0 , 0}$ \\
\hline
\end{tabular}

En relación al número de intentos de punción la media fue de 3,1 $\pm 2,5$ veces (Tabla 2).

Otros estudios presentaron una variación en esta media de 2,7 a 3,4 tentativas, sin embargo con éxito en la primera tentativa entre 21,6 a $47,0 \%$, superior a la encontrada en este estudio $^{(7,10)}$. Uno de estos estudios señala que los catéteres insertados tras más de un intento de punción, presentaron dificultad de progresión y los vasos de primera opción tenían el calibre muy fino. Tales dificultades en la inserción están directamente ligadas al número de punciones ${ }^{(10)}$. (Tabla 2)

Tabela 2 - Distribuição da freqüência e percentagem segundo o número de tentativas de inserção do cateter PICC. Uberaba (MG) -2009.

\begin{tabular}{lcc}
\hline Número de tentativas & $\mathbf{N}=\mathbf{5 5}$ & $\%$ \\
\hline Uma tentativa & 9 & 16,4 \\
Duas tentativas & 15 & 27,3 \\
Três tentativas & 9 & 16,4 \\
Quatro tentativas & 7 & 12,7 \\
Cinco tentativas & 1 & 1,8 \\
Seis ou mais & 5 & 9,1 \\
Não informado & 9 & 16,3 \\
\hline TOTAL & $\mathbf{5 5}$ & $\mathbf{1 0 0 , 0}$ \\
\hline
\end{tabular}


La localización inicial de la punta del catéter fue verificada mediante examen radiológico y en $21(38,2 \%)$ se encontraba centralmente localizada (Tabla 3).

Otros estudios describieron la localización inicial central variando de 25,9\% a 40,9\%. El alto índice de PICC de localización intracardíaca indica un posible fallo en la medida realizada antes de la inserción, y en estos casos es necesaria la retirada del catéter. Catéteres intracardíacos y periféricos tienen 0,33 veces más posibilidades de presentar complicaciones en comparación con los catéteres centrales ${ }^{(6,10)}$.

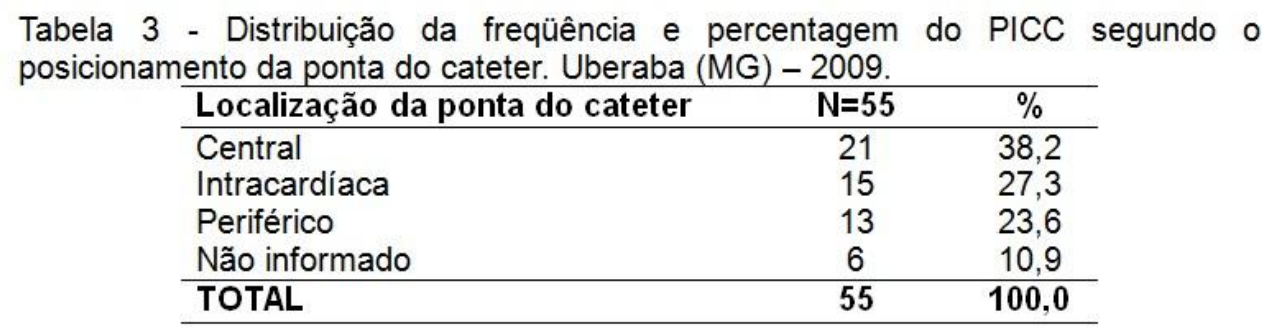

En cuanto a los motivos que llevaron a la retirada del PICC, 17(30,9\%) fueron por obstrucción del catéter (Tabla 4).

La oclusión es definida como la obstrucción parcial o completa del catéter que impide o dificulta la aspiración de sangre, llevando a la pérdida de la permeabilidad del catéter. La incidencia de este tipo de complicación varía entre 2 y $44 \%{ }^{(18,19)}$.

Las principales causas de oclusión de estos catéteres son las mecánicas (dobladas o compresión del lumen del catéter), la obstrucción trombótica causada por la formación de coágulos interna o externamente al catéter y obstrucción no trombótica consecuente con la cristalización intralumial de medicamentos incompatibles o nutrición parenteral ${ }^{(18,19,20)}$.

La incidencia de ruptura de estos catéteres (PICC), varía entre 4 y $5 \%$. Los catéteres de pequeño calibre, especialmente de silicona, pueden quebrarse o romperse fácilmente si son manipulados de forma inadecuada y romperse con la presión ejercida por la utilización de jeringas menores de $10 \mathrm{ml}^{(21)}$.

Tabela 4 - Distribuição dos motivos de remoção do cateter PICC. Uberaba (MG) 2009.

\begin{tabular}{lcc}
\hline Motivos de remoção do PICC & $\mathbf{N = 5 5}$ & $\%$ \\
\hline Obstrução & 17 & 30,9 \\
Término da terapia intravenosa & 15 & 27,3 \\
Ruptura & 9 & 16,4 \\
Extravasamento & 3 & 5,5 \\
Exteriorização acidental & 5 & 9,1 \\
Infecção & 2 & 3,6 \\
Flebite & 2 & 3,6 \\
Falso trajeto & 1 & 1,8 \\
Não informado & 1 & 1,8 \\
\hline TOTAL & $\mathbf{5 5}$ & $\mathbf{1 0 0 , 0}$ \\
\hline
\end{tabular}


En cuanto al tiempo de permanencia del PICC se observó que la media fue de 8,8 \pm 6,1 días (mínimo 2 horas y máximo 33 días). Destaca en este estudio, que el catéter que presentó tiempo de permanencia de solo 2 horas fue insertado en vena retroauricular tras 15 tentativas y presentó falso trayecto siendo por este motivo retirado. La mayoría de los catéteres permanecieron insertados de seis a diez días (Tabla 5).

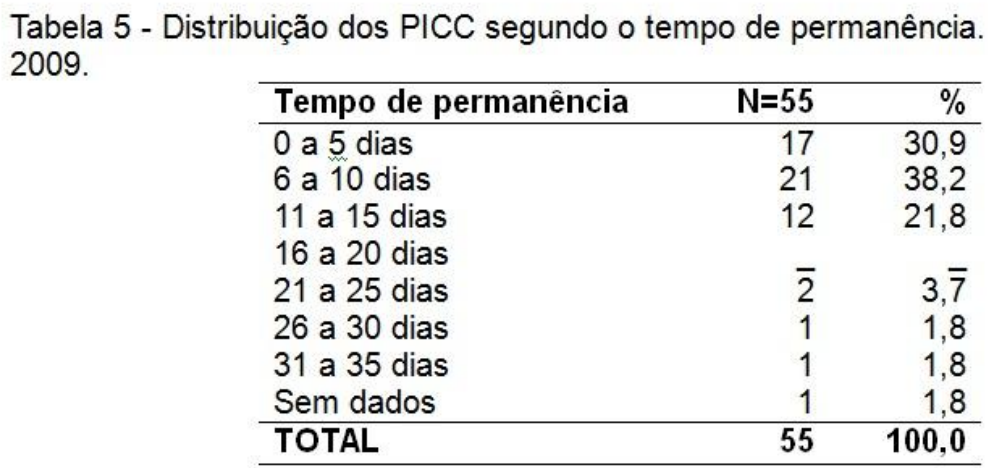

Los catéteres PICC pueden permanecer por períodos indeterminados de tiempo, siempre que se realice el cuidado apropiado y no aparezcan complicaciones. Hay estudios que reportan un tiempo de permanencia de 10 a 73 días $^{(22)}, 13$ a 19 días $^{(9)}$ y 11 a 32 días $^{(23,24)}$.

En esta investigación, se verificó el periodo de permanencia del catéter PICC inferior a los encontrados en la literatura ${ }^{(9,22-24)}$.

Tras la retirada del PICC, 38(69,1\%) fueron sometidos a cultivo de punta de catéter, $10(18,2 \%)$ no realizaron cultivo y $7(12,7 \%)$ no hubo registro de pedido de análisis microbiológico. Los resultados del análisis microbiológico evidenciaron en solo $1(1,8 \%)$ el desarrollo del hongo, Candida parapsilosis.

En cuanto a las complicaciones, la mayoría $35(85,5 \%)$ no presentó complicaciones relacionadas con el PICC. Entre las complicaciones identificaron 2 (4,9\%) casos de flebitis (Tabla 6). Es importante destacar que en los casos de sepsis fúngica y bacteriana no hubo confirmación de la relación con el uso del PICC, ya que los RN fueron sometidos a otros procedimientos considerados factores de riesgo para la ocurrencia de estas complicaciones, entre ellas el uso de catéter umbilical, y no había en los prontuarios analizados registro en cuanto al origen del foco infeccioso.

Según estudios, la flebitis mecánica es una de las complicaciones más observada en pacientes en uso de PICC, variando entre 5 y $26 \%$ y son evidentes a partir de 48 a 72 horas después de la inserción o retirada del dispositivo ${ }^{(21,25,26)}$.

En esta pesquisa, la ocurrencia de sepsis, tanto fúngica como bacteriana, corrobora con lo mostrado en otros estudios. Hay estudios que señalan la incidencia de sepsis relacionada con el PICC que puede variar entre 2 y $21 \%{ }^{(18,25,27)}$. Sin embargo, hay también evidencias que apuntan a que el $80 \%$ de los casos de bacteremias identificados en hospitales están causados por catéteres venosos centrales, y de estos, 1 a $3 \%$ corresponden a aquellos debidos al uso del PICC $^{(27)}$.

Hay también otros estudios que señalan la prematuridad, o bajo peso, uso de nutrición parenteral prolongada y la ocurrencia de infecciones maternas durante la gestación como 
factores que acentúan el riesgo para ocurrencia de infección de corriente sanguínea relacionada con el uso del catéter venoso central ${ }^{(28)}$.

\section{CONCLUSIONES}

El estudio evidencia que el PICC en la UTI neonatal, representa un recurso terapéutico importante en la asistencia de enfermería, por eso el equipo de enfermeros debe estar capacitado para garantizar que el lugar de inserción sea escogido adecuadamente, que el procedimiento sea ejecutado con seguridad, disminuyéndose el número de tentativas de punción, que el catéter sea manipulado correctamente evitando principalmente las complicaciones como obstrucción e infección y que el catéter sea de hecho un recurso de acceso intravenoso de larga permanencia.

Los resultados sugieren que debe haber inversión en programas de formación y educación permanente, establecimiento de protocolo de cuidados, y realización de estudios longitudinales que permitan un mejor acompañamiento de la ocurrencia de las complicaciones relacionadas con el uso de este tipo de catéter.

\section{REFERENCIAS}

1. D'elia C, Correira MS, Oliveira SD, Barbosa NMM. Fístula broncovascular: complicação de cateter venoso central percutâneo em neonato. J. Pediatr. 2002;78(4):347-50.

2. Secoli SR, Jesus VCJ. Complicações acerca do Cateter Venoso Central de Inserção Periférica (PICC). Cienc. Cuid. Saúde. 2007;6(2):252-60.

3. Vendramim PP, Pedreira MLG, Peterlini MAS. Cateteres centrais de inserção periférica em crianças de hospitais do município de São Paulo. Rev. Gaúcha Enferm. 2007;28(3):33139.

4. Rodrigues ZS, Chaves EMD, Cardoso MVLML. Atuação do enfermeiro no cuidado com o cateter central de inserção periférica no recém-nascido. Rev. Bras. Enferm. 2006;59(5): 62629.

5. Lourenço SA, Kakehashi TY. Avaliação do cateter central de inserção periférica em neonalologia. Acta Paul. Enf. 2003;16(2):26-32.

6. Camargo PP, Kimura AF, Toma E, Tsunechiro. Localização inicial da ponta de cateter central de inserção periférica (PICC) em recém-nascidos. Rev. Esc. Enferm. USP. 2008; 42(4):723-28.

7. Camargo PP. Procedimento de Inserção, manutenção e remoção do cateter de inserção periférica [dissertação]. São Paulo: Universidade de São Paulo; 2007.

8. National Nosocomial Infections Surveillance System. National Nosocomial Infections Surveillance (NNIS) System report, data summary from January 1992 through June 2004, issued October 2004. Am. J. Infect. Control. 2004;32:470-85.

9. Miranda MA. Epidemiologia do uso de cateter venosos central em unidade de Terapia Intensiva Neonatal. [dissertação]. Belo Horizonte: Universidade Federal de Minas Gerais; 2005.

10. Braga LM. Cateter Central de Inserção Periférica-CCIP: investigação prospectiva em recém-nascidos submetidos á terapia intravascular [dissertação]. Belo Horizonte: Instituto de Previdência dos Servidores do Estado de Minas Gerais; 2006.

11. Tamez RN, Silva MJP. Enfermagem na UTI neonatal: assistência ao recém-nascido de alto risco. Rio de Janeiro: Guanabara Koogan; 2006.

12. Kenner C. Enfermagem Neonatal. Rio de Janeiro: Reichmamn Editores; 2001. 
13. Centers for Diesase Control and Prevention. Departament of Health and Human Services. Intravascular device: related infections preventions; guidelines availability: notice. Atlanta (GO): CDC; 2002.

14. Richtmann R, Silva LPS, El-Far F, Amarante JMB, Kawagoe JY, Konkewicz LR, et al. Diagnóstico e prevenção das infecções hospitalares em neonatologia. São Paulo: APECIH; 2002.

15. Chaves EMC. Redução da infecção da corrente sangüínea através do filtro bacteriológico em prematuros [dissertação]. Fortaleza: Universidade Estadual do Ceará; 2003.

16. Herrera CR, Mayor SJ, Vasquez TML. El cateter venoso percutâneo: uma opcion económica y segura para niños pretérmino de muy bajo peso. Colômbia Méd. 1996;27: 11 51.

17. Romaniello HO. Evoluación Del empleo de los cateteres percutâneos em ricién nascidos. Arch. Argent. Pediatr. 1999;97(2): 82-85.

18. Philpot $P$, Griffiths V. The peripherally inserted central catheter. Nurs. Stand. 2003;17(44):39-49.

19. Farjo L. Blood collection from peripherally inserted central venous catheters: an institution's effort to evaluate and update its current policy. J. Infus. Nurs. 2003;26(6):374-79.

20. Secoli SR, Kishi HM, Carrara D. Inserção e manutenção do PICC: aspectos da prática clínica de enfermagem em oncologia. Prática Hospitalar. 2006;7(47):155-62.

21. Gorski LA, Czaplewski LM. Peripherally Inserted Central Catheters and Midline Catheters for the Homecare Nurse. J. Infus. Nurs. 2004;27(6):399-409.

22. Pimiento SE. La cateterización venosa central en La Fundación Santa Fé de Bogotá: Do seguimiento Asistencial a indicador de Gestión. Revista Medicina. 2002;24(60):169-87.

23. Chathas MK, Paton JB. Sepsis outcomes in infants and children with central venous catheters: Percutaneous versus surgical insertion. J. Obstet. Gynecol Neonatal Nurs. 1996;25(6):500-06.

24. Shulman RJ, Pokorny WJ, Martin CG, Petitt R, Baldaia L, Roney D. Comparison of Percutaneous and surgical placement of central venous catheters in neonates. J. Pediatr. Surg. 1986;21(4):348-50.

25. Griffiths VR, Philpot P. Peripherally inserted central catheters (PICC): do they have a role in the care of the critically ill patient? Intensive Crit. Care. Nurs. 2002;18(1):37-47.

26. Gabriel J. PICC securement: minimizing potential complications. Nurs. Stand. 2001;15(43):42-44.

27. Major BM, Crow MM. Peripherally inserted central catheters in the patient with cardiomyopathy: the most costeffective venous access. J. Intraven. Nurs. 2000;23(6):366-70. 28. Barbosa MH, Figueiredo VR, Pires OS. Infecção de corrente sanguínea relacionada ao uso de Cateteres Venosos Centrais em Recém-Nascidos. Nursing. 2009;11(129):82-86.

ISSN 1695-6141

@) COPYRIGHT Servicio de Publicaciones - Universidad de Murcia 